\title{
A Linear Matrix Inequality Approach to Robust Damping Control Design in Power Systems with Superconducting Magnetic Energy Storage Device
}

\author{
Bikash C. Pal, Alun H. Coonick, Imad M. Jaimoukha, and Haitham El-Zobaidi
}

\begin{abstract}
This paper presents a systematic design procedure for a robust damping controller based on the Linear Matrix Inequality (LMI) approach employing a Superconducting Magnetic Energy Storage (SMES) device. The design procedure takes advantage of the multi-objective features of LMI based design techniques. The procedure is applied to enhance the damping of inter-area oscillations in a two-area four-machine test system.
\end{abstract}

Index Terms $-H_{\infty}$, inter-area oscillation, linear matrix inequalities (LMI), magnetic energy storage, model reduction, power system damping controller, robust control, superconducting.

\section{INTRODUCTION}

$\mathbf{T}$ HE DAMPING of inter-area oscillations in power systems has been an important topic of research for several decades [1]-[3]. In the last few years, the concept of $I_{\infty}$ control design to guarantee robust performance and robust stability in the face of uncertainty in plant parameter variation has been applied in power systems to damp out low frequency oscillations [4]-[6]. Normally the problem is formulated as a weighted mixed sensitivity design. The Riccati based solution to this problem always gives rise to a controller that suffers from pole-zero cancellations of the plant and the controller [7]. Although the poorly damped inter-area mode does not appear between the controller input and plant output, it may appear in some other system outputs [4], if the weights are not chosen carefully. The implications of this in a power system is that the inter-area mode may not be damped at all. In addition to this, a standard Riccati based solution to a mixed sensitivity formulation fails to provide a solution when the augmented plant has invariant zeros either at infinity or on the imaginary axis. Furthermore, some of the specifications in the time domain, such as settling time, peak overshoot (closed-loop damping ratio) cannot be captured in a straightforward way in a Riccati based design. In power system damping control design, the main objective is to improve the damping ratio of the electromechanical modes over widely varying operating conditions. This type of objective cannot be easily translated into suitable weighting functions that can augment the plant and therefore provide a satisfactory controller.

The $H_{\infty}$ controller design based on the LMI formulation with a regional pole placement objective can address this type of problem in a more tractable way. The solution to the LMI based

Manuscript received July 28, 1998; revised October 27, 1998.

The authots are with the Department of Electrical and Electronic Engineering, Imperial College, London, SW7-2BT, U.K. (e-mail:\{b.pal; a.coonick\}@ic.ac.uk; (jaimolka; zobaidih\}@ps.ic.ac.uk).

Publisher Item Identifier \$ 0885-8950(00)01895-2.

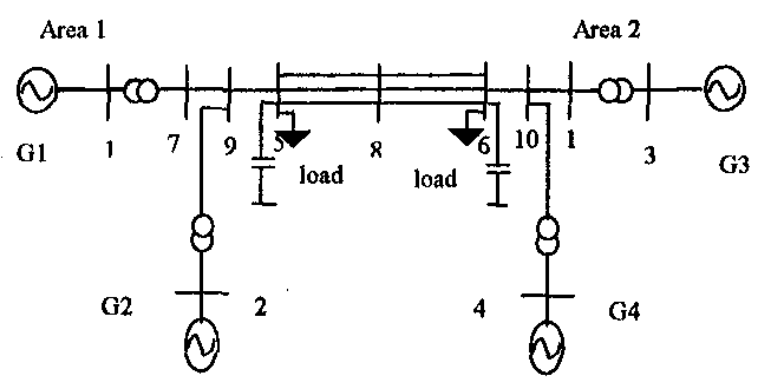

Fig. 1. A four machine test system.

formulation does not require the plant to be of a special class. The plant can have invariant zeros anywhere in the $s$-plane. This feature of LMI makes weight selection far more easier than the Riccati based $H_{\infty}$ design.

Since the early seventies, superconducting magnetic energy storage (SMES) systems have been suggested by many researchers [8]-[10] for the damping of power system oscillations. The damping of real power oscillations is more effective through real power modulation. Since an SMES unit can provide real power, it can damp out power oscillations very effectively when its output is modulated by the controller. The SMES is considered as a damping control device here.

This paper consists of seven sections. Following this introduction, Section II describes a study system and a low frequency model of the SMES. The best location for the SMES in the study system is obtained by a residue method [11]. In Section III, the mixed sensitivity design with regional pole placement constraints in the LMI framework is discussed. Section IV deals with the design of the power system damping controller based on the approach discussed in Section III. The performance of the controller is assessed in the frequency and time domains in Section V. The effects of load characteristics on the closed loop damping ratios are also discussed in Section V. The sizing of the SMES is discussed in Section VI. Section VII concludes the paper.

\section{STUDY SYSTEM}

A two-area four-machine test system, as shown in Fig. 1, is considered. This system has been studied by many researchers [3], [5], [8] for inter-area mode damping improvement. Several operating points (OP) for various levels of power transfer and transmission strength variations are listed in Appendix A. The load is considered to be of a constant impedance type. The 

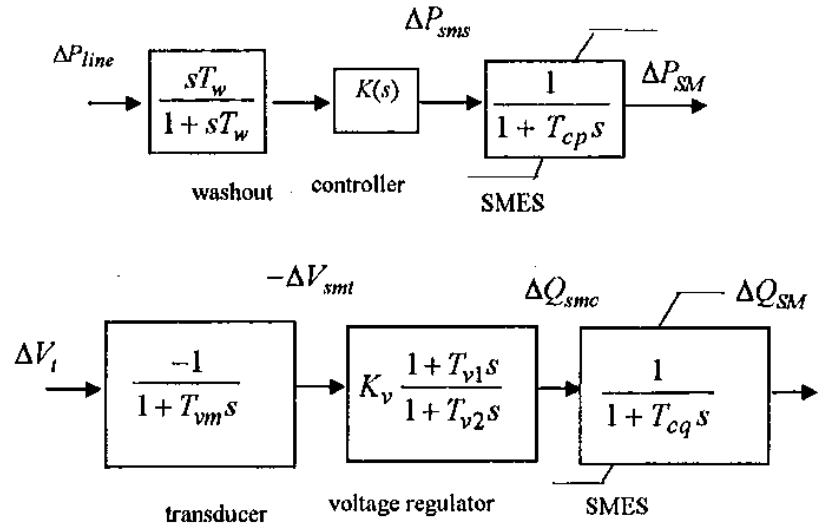

Fig. 2. Small signal model of a SMES unit.

power flow is created by an uneven split in loads and generations. Two capacitor banks, injecting $200 \mathrm{Mvar}$ each, on bus \#5 and bus \#6 are assumed to facilitate power flow. The response of the SMES is assumed to be very fast and is modeled by a single time constant [8]. The SMES is equipped with a fast acting voltage regulator for which the small signal model is shown in Fig. 2. The various parameters of the generators, exciters, and transmission system can be found in [3] and the parameters for the SMES are listed in Appendix A. For a power flow of $400 \mathrm{MW}$ from area 1 to area 2, an eigenvalue analysis of the system is performed in the open-loop. Three electromechanical modes (mode 1, mode 2 and mode 3 ) are found with damping ratio/frequency of oscillations to be $0.009 / 0.69,0.085 / 1.14$ and $0.115 / 1.15 \mathrm{~Hz}$, respectively. Further examination of the modes shows that mode 1 involves all of the four generators. So it is an inter-area mode. The last two modes arc intraplant modes of areas 1 and 2, respectively. In this paper, we restrict ourselves to the design of the damping controller for the inter-area mode.

The siting of the SMES in the system is determined on the basis of its stabilizing potential to the inter-area mode. The technique adopted here is based on the residue method proposed by Martin et al. [11]. Various locally available signals, e.g., bus voltage, bus frequency, real power in all of the lines terminated on a bus, line current magnitudes, rotor speed, etc., are considered as the input signals. Observability analysis shows that the active line power, active line current, and line current magnitude carry valuable information about the inter-area mode. The normalized residues at each bus for the most effective stabilizing signal are computed and are displayed in Fig. 3 for OP5 and OP7. It is found that when power flows from area 1 to area 2, bus \#10 is the most effective location with the input signal taken from the power flow in the line between bus\#10 and bus\#6. For power flow from area 2 to area 1 , bus $\# 9$ is the best location with active component of the power in the line between bus\#9 and bus\#5 as the best signal, So the best location for the SMES is dependent on the power flow pattern. It is decided to place the SMES at bus\#10 assuming that power flows most of the time from area 1 to area 2. It is also interesting to see that bus\#8, i.e. middle of the tie lines, is the one with least residue. This suggests that the mid point of the long distance transmission

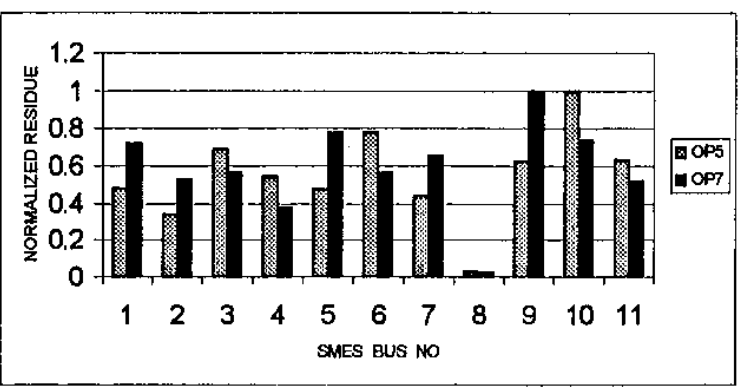

Fig. 3. Transfer function residue at various busses.

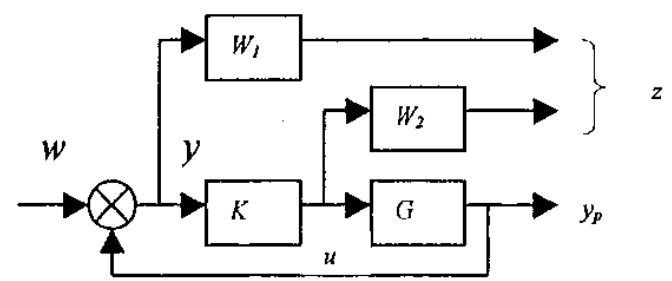

Fig. 4. Standard mixed sensitivity feedback configuration.

system is not effective for damping control through real power modulation.

\section{ROBUST CONTROL-AN LMI APPROACH}

The theory of LMI is rather involved and detail can be found in [12]. The design problem treated in this paper consists of finding an internally stabilizing controller that satisfies an infinity norm constraint, whilc cnsuring that the closed-loop poles lie in certain locations in the complex plane. This section describes the control problem in more detail and gives an LMI formulation for each of these design specifications. Consider the standard mixed sensitivity configuration of Fig. 4.

$G$ is the open loop plant, $K$ is the controller to be designed and $W_{1}$ and $W_{2}$ are weights. This design would minimize a weighted mix of the transfer function $S=(I-G K)^{-1}$ that ensures disturbance attenuation and good tracking; and $K S=$ $K(I-G K)^{-1}$ that handles the robustness issues and constrains the controller effort. This mixed sensitivity $(S / K S)$ design objective is represented as

$$
\left\|\begin{array}{c}
W_{1} S \\
W_{2} K S
\end{array}\right\|_{\infty} \leq \gamma
$$

where $\gamma$ is the bound on $H_{\infty}$ norm.

Details of the mixed sensitivity control design can be found in [13]. The state space description of the system above can be written as:

$$
\begin{aligned}
\dot{x} & =A x+B_{1} u+B_{2} u \\
z & =C_{1} x+D_{11} u+D_{1.2} u \\
y & =C_{2} x+D_{2} w+D_{22} u .
\end{aligned}
$$

The controller $K$ can also be described in state space form

$$
\dot{x}_{k}=A_{k} x_{k}+B_{k} y
$$




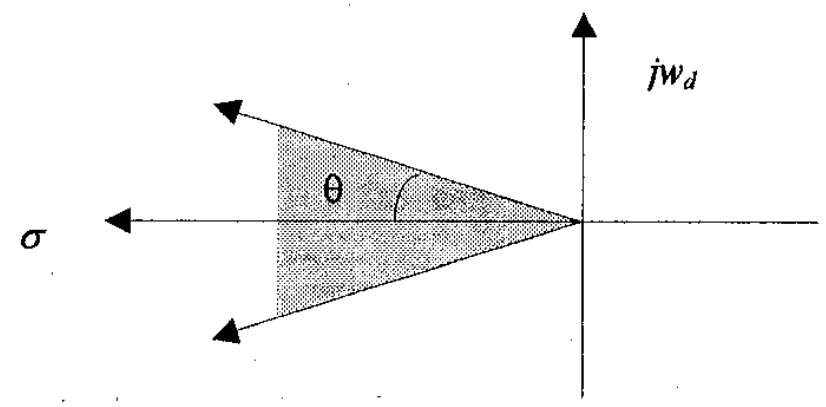

Fig. 5. Region for the closed loop poles with minimum damping ratio cos $\theta$.

$$
u=C_{k} x_{k}+D_{k} y \text {. }
$$

$D_{22}$ is dependent on the input-output relationship of the plant. For the small signal model of the SMES described earlier in Section II with line power as the stabilizing feedback signal, the plant becomes strictly proper resulting in $D_{22}=0$. Other FACTS devices with a specific choice of the stabilizing feedback signal may result in a nonzero $D_{22}$. In those cases the design can be carried out considering the plant as strictly proper (setting $D_{22}=0$ ) and the resulting controller, $K$ can be modified later to include the effect of $D_{22}$ by $\hat{K}=K\left(1-D_{22} K\right)^{-1}$ [14]. So without loss of generality, $D_{22}$ can be set to zero making the derivation simpler.

The transfer function between " $w$ " and " $z$ " can be described as

$$
T_{z w}=\left\|\begin{array}{c}
W_{1} S \\
W_{2} K S
\end{array}\right\|=C_{c l}\left(s I-A_{c l}\right)^{-1} B_{c l}+D_{c l}
$$

where

$A_{c l}=\left[\begin{array}{cc}A+B_{2} D_{k} C_{2} & B_{2} C_{k} \\ B_{k} C_{2} & A_{k}\end{array}\right] ; B_{c l}=\left[\begin{array}{c}B_{1}+B_{2} D_{k} D_{21} \\ B_{k} D_{21}\end{array}\right]$

$C_{c l}=\left[\begin{array}{ll}C_{1}+D_{12} D_{k} C_{2} & D_{12} C_{k}\end{array}\right] ; D_{c l}=D_{1.1}+D_{12} D_{k} D_{21}$

The objective of the mixed sensitivity minimization is to find an internally stabilizing controller that minimizes

$$
\left\|\begin{array}{c}
W_{1} S \\
W_{2} K S
\end{array}\right\|_{\infty}
$$

In an LMI formulation [15], the same objective is achieved in the sub-optimal sense if there exists an $X_{\infty}=X_{\infty}^{T}>0$ such that

$$
\left(\begin{array}{ccc}
A_{c l}^{T} X_{\infty}+X_{\infty}+A_{c l} & B_{c l} & X_{\infty} C_{c l}^{T} \\
B_{c l}^{T} & -I & D_{c l}^{T} \\
C_{c l} X_{\infty} & D_{c l} & -\gamma^{2} I
\end{array}\right)<0
$$

with $\left\|C_{c l}\left(s I-A_{c l}\right)^{-1} B_{c l}+D_{c l}\right\|_{\infty}<\gamma ;$ and the controller is said to be $\gamma$ sub-optimal.

It is known that the transient response of a linear system is related to the location of its poles. For example, the step response of a second-order system with poles $\lambda=-\zeta \omega_{n} \pm j \omega_{d}$ is fully characterized in terms of the undamped natural frequency $\omega_{n}=|\lambda|$, the damping ratio $\zeta$, and the damped natural frequency $\omega_{d}$. Acceptable transient response can be achieved by placing all of the closed-loop poles inside a conic sector as shown in Fig. 5.

The damping ratio of all the poles lying inside the sector is guaranteed to have minimum damping ratio $\zeta=\cos \theta$.
The LMI characterization of the conical sector is straightforward. It is shown in [16] that the state matrix, $A_{c l}$ of the closed loop system, has all its poles inside this conical sector if and only if there exists $X_{p}=X_{p}^{T}>0$ such that

$$
\left[\begin{array}{l}
\sin \theta\left(A_{c l} X_{p}+X_{p} A_{c l}^{T}\right) \cos \theta\left(A_{c l} X_{p}-X_{p} A_{c l}^{T}\right) \\
\cos \theta\left(X_{p} A_{c l}^{T^{\prime}}-A_{c l} X_{p}\right) \sin \theta\left(A_{c l} X_{p}+X_{p} A_{c l}^{T}\right)
\end{array}\right]<0 .
$$

This can be expressed in Kronecker product form [14]

$$
\left[\eta \otimes A_{c l} X_{p}+\eta^{\prime} \otimes X_{p} A_{c l}^{T}\right]<0
$$

where

$$
\eta=\left(\begin{array}{rr}
\sin \theta & \cos \theta \\
-\cos \theta & \sin \theta
\end{array}\right)
$$

The two inequalities in (7) and(9) are not jointly convex as they can admit two different solutions $\left(X_{\infty} \neq X_{p}\right)$. In order to enforce joint convexity, a common solution, $X_{\infty}=X_{p}=$ $X$, can be sought with a certain degree of conservatism. The problem therefore reduces to minimization of $\gamma$ under $H_{\infty}$ and the pole placement constraints. Inequalities (7) and (9) contain $A_{c l} X, C_{c l} X$, i.e. products of $X$ and the controller variables. The resulting problem is therefore nonlinear and so it cannot be handled by LMI optimization directly. To make it linear, a change of controller variables is necessary. This is performed in $[16]$ and $[17]$ to give the following simplified LMI's:

$$
\begin{gathered}
\left(\begin{array}{cc}
R & I \\
I & S
\end{array}\right)>0 \\
{\left[\begin{array}{cc}
\Psi_{11} & \Psi_{21}^{T} \\
\Psi_{21} & \Psi_{22}
\end{array}\right]<0} \\
{\left[\eta \otimes \Phi+\eta^{T} \otimes \Phi^{T^{\prime}}\right]<0,}
\end{gathered}
$$

The variables $\Psi_{11}, \Psi_{12}, \Psi_{21}, \Psi_{22}$, and $\Phi$ are given in terms of new controller variables $\hat{A}, \hat{B}, \hat{C}, \hat{D}$, and the derivation of the LMI's (10)-(12) is given in Appendix B. It is interesting to note that the LMI formulation of the weighted mixed sensitivity approach in addition to pole placement objective is appealing from a practical point of view. The LMI in (11) addresses disturbance attenuation, tracking, constraints on control effort, and robustness aspects of the control design. The LMI in (12) guarantees a minimum closed-loop damping ratio of $\cos \theta$. The LMI's in (10)-(12) can be solved for $\hat{A}, \hat{B}, \hat{C}$, and $\hat{D}$ by recently developed interior-point optimization algorithms [18]. Once $\hat{A}, \hat{B}, \hat{C}$, and $\hat{D}$ are found, the actual controller variables $A_{k}, B_{k}, C_{k}$, and $D_{k}$ can be recovered from $\hat{A}, \hat{B}, \hat{C}$, and $\hat{D}$ by solving (13)-(16) in Appendix B.

\section{Power System DAMPing Controller}

The above formalism is applied to the two-area test system as described in Section II. The OP5 is taken as the nominal operating case and the transfer function of the plant $G$ is obtained. The original plant is of 32 nd order and is reduced to 4 th order by the balanced singular perturbation [19] technique. Following the standard guidelines of a mixed sensitivity design [13], weights $W_{1}$ and $W_{2}$ are chosen as low and high pass filters, respectively. The controller is designed and when it is tested works well for the reduced (4th order) system. When it is applied to the full (32nd order) system, the open loop gain in the low frequency 
TABILE I

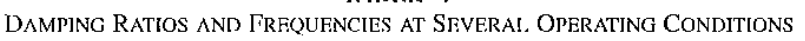

\begin{tabular}{|c|c|c|c|c|c|c|c|c|c|c|c|c|c|}
\hline \multirow{3}{*}{$\begin{array}{l}\text { Power flow } \\
\text { scenario }\end{array}$} & \multirow{3}{*}{$\begin{array}{l}\text { No of } \\
\text { ties }\end{array}$} & \multicolumn{6}{|c|}{ Open loop damping ratios and frequencies } & \multicolumn{6}{|c|}{ Closed toop damping ratios and frequencies } \\
\hline & & \multicolumn{2}{|c|}{ Mode 1} & \multicolumn{2}{|c|}{ Mode 2} & \multicolumn{2}{|c|}{ Mode 3} & \multicolumn{2}{|c|}{ Mode 1} & \multicolumn{2}{|c|}{ Mode 2} & \multicolumn{2}{|c|}{ Mode 3} \\
\hline & & \multicolumn{2}{|r|}{$(\mathrm{Hz})$} & \multicolumn{2}{|c|}{$\varsigma \quad f(\mathrm{~Hz})$} & \multicolumn{2}{|c|}{$\varsigma \quad f(\mathrm{~Hz})$} & \multicolumn{2}{|c|}{ 与. $f(\mathrm{~Hz})$} & \multicolumn{2}{|c|}{$\varsigma \quad f(\mathrm{~Hz})$} & \multicolumn{2}{|c|}{$\varsigma \quad f(\mathrm{~Hz})$} \\
\hline OP1 & 3 & 0.026 & 0.72 & 0.121 & 1.12 & 0.114 & 1.16 & 0.215 & 0.72 & 0.119 & 1.12 & 0.147 & 1.13 \\
\hline OP2 & 2 & 0.019 & 0.64 & 0.122 & 1.12 & 0.115 & 1.16 & 0.172 & 0.64 & 0.121 & 1.12 & 0.141 & 1.13 \\
\hline OP3 & 3 & 0.010 & 0.71 & 0.115 & 1.15 & 0.085 & 1.15 & $\mathbf{0 . 2 1 0}$ & $\mathbf{0 . 7 0}$ & 0.148 & 1.12 & 0.084 & 1.14 \\
\hline OP4 & 2 & 0.005 & 0.62 & 0.116 & 1.15 & 0.085 & 1.14 & 0.172 & 0.62 & 0.142 & 1.12 & 0.085 & 1.14 \\
\hline$\overline{\mathrm{OP} 5}$ & 3 & 0.009 & 0.69 & 0.115 & 1.15 & 0.085 & 1.14 & 0.216 & 0.69 & 0.146 & 1.12 & 0.084 & 1.14 \\
\hline OP6 & 2 & 0.003 & 0.59 & 0.114 & 1.14 & 0.085 & 1.14 & 0.179 & 0.58 & 0.138 & 1.12 & 0.085 & 1.14 \\
\hline OP7 & 3 & 0.009 & 0.69 & 0.119 & 1.12 & 0.082 & 1.17 & 0.145 & 0.72 & 0.118 & 1.12 & 0.117 & 1.15 \\
\hline OP8 & 2 & 0.002 & 0.58 & 0.119 & 1.11 & 0.080 & 1.17 & 0.103 & 0.59 & 0.119 & 1.11 & 0.106 & 1.15 \\
\hline
\end{tabular}

range $(0.01-0.1 \mathrm{~Hz})$ is found to be very high and makes the overall closed-loop system unstable. In view of this, the weight $W_{2}$ is modified to have a high gain in the low frequency range thus reducing the gain of the controller in this range. The modified weights $W_{1}$ and $W_{2}$ are given by

$$
W_{1}(s)=\frac{1}{s+1.12} ; W_{2}(s)=\frac{0.4924 s^{2}+1.956 s+4.9362}{s^{2}+0.002 s}
$$

In all of these attempts, it is found that minimization of $H_{\infty}$ norms [i.c., LMI in (10) and (11)] alone does not provide an adequate damping ratio in the closed-loop. The pole placement objective [LMI in (12)] when added provides the desired damping. A value of $\theta=81.37^{\circ}$ is used to define the conical sector to ensure a minimum damping ratio of 0.15 for the closed-loop system. The controller is designed by solving the LMI's with the help of the Matlab LMI toolbox [20] and is originally of 7th order. It is reduced to 2 nd order by the balanced singular perturbation technique. The robust control toolbox [21] in Matlab is used for the model reduction task. A washout filter of $10 \mathrm{~s} \mathrm{time}$ constant is added to further eliminate any steady-state bias in the damping controller output. The controller with the washout filter is given by

$$
K(s)=\frac{10 s}{1+10 s}\left(-0.8178 \frac{s^{2}+8.35 s}{s^{2}+3.85 s+51.02}\right) .
$$

\section{Performance Evaluations}

The inter-area mode damping in the closed-loop is examined for various power flow conditions, as described in Appendix A, of the system. Full order systems are considered in all of the operating conditions to compute the damping. Table $I$ shows these results for the various power flow scenarios. It can be seen that the damping is very good in all of the operating conditions. When no power flows, the damping ratios are 0.215 and 0.172 for the pre-fault (OP1) and the post-fault (OP2) cases, respectively. The damping ratios are 0.210 (OP3) and 0.172 (OP4) when $200 \mathrm{MW}$ power flows from area 1 to area 2 . When the power flow is further increased to $400 \mathrm{MW}$ in the same direction, the damping ratio in the pre-fault (OP5) and the postfault (OP6) conditions are 0.216 and 0.179 respectively. This indicates that the controller is robust in the range $0-400 \mathrm{MW}$ of power flow variation from area 1 to area 2 . When $400 \mathrm{MW}$ of flow is attempted from area 2 to area 1 , it is found that the damping ratio in the pre-fault (OP7) and post-fault (OP8) conditions are 0.145 and 0.103 and are considered to be adequate.

The performance of the controller is evaluated in the face of various load characteristics. Load characteristics of constant current (CC), constant power (CP), and an equal mix of constant current $(50 \%)$ and constant power $(50 \%)$ are considered. The damping ratio and frequency of the inter-area mode for the open-loop and closed-loop are computed for various operating conditions and are displayed in Table II. It can be seen from the results in Table II that the controller ensures very good damping for various load characteristics.

In order to examine the nature of the interaction with other modes, the damping ratios and frequencies of those modes are computed and are displayed in Table I. It can be seen that the damping of these modes is virtually unaffected by the controller.

To verify the performance of the controller in the face of system nonlinearities, saturation, and controller output limit, nonlinear simulation is carried out for 20 seconds. A three-phase fault near bus $\# 8$ on one of the tie lines is created for $70 \mathrm{~ms}$ followed by the opening of the faulted line. OP5 is considered as the pre-fault case. The variations of angles of G1 and G2 with respect to G3 are plotted for the last $15 \mathrm{~s}$ of the simulation in Fig. 6. The power flow through the line and the action of the controller is also displayed in Fig. 6. It is clear from the plots that the inter-area mode is well damped by the controller, which is otherwise very poorly damped. The response of the controller shows that initially for a few seconds the controller output reaches its limits of \pm 0.1 . p.u. After this period it regains linearity and damps out the oscillation very quickly. The performance of the controller is further verified for another operating point OP7. In OP7 the direction of power flow is different from that in OP5. The dynamic behavior of the same set of variables as in Fig. 6 for the same disturbance is computed and is plotted in Fig. 7. It can be seen that the controller eventually damps out the oscillation in 10-12 $\mathrm{s}$. This clearly justifies the robust property of the controller taking system nonlinearities into account. 
TABLE II

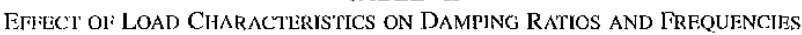

\begin{tabular}{|c|c|c|c|c|c|c|c|c|c|c|c|c|c|}
\hline \multirow{2}{*}{$\begin{array}{l}\text { Power flow } \\
\text { scenario }\end{array}$} & \multirow{2}{*}{$\begin{array}{c}\text { No of } \\
\text { ties }\end{array}$} & \multicolumn{6}{|c|}{ Open loop damping ratios and frequencies } & \multicolumn{6}{|c|}{ Closed loop damping ratios and frequencies } \\
\hline & & $\overline{\mathrm{CC}}$ & $\mathrm{Hz})$ & & $\mathrm{Hz})$ & & $\mathrm{CC}+\mathrm{CP}$ & $\varsigma$ & $\mathrm{Hz})$ & & Hz) & $\mathrm{CO}$ & $\begin{array}{l}\mathrm{CP} \\
\mathrm{Hz})\end{array}$ \\
\hline OP1 & 3 & 0.029 & 0.73 & 0.031 & 0.74 & 0.030 & 0.73 & 0.221 & 0.72 & 0.228 & 0.73 & 0.225 & 0.73 \\
\hline OP2 & 2 & 0.023 & 0.65 & 0.026 & 0.66 & 0.024 & 0.66 & 0.180 & 0.64 & 0.189 & 0.65 & 0.184 & 0.65 \\
\hline OP3 & 3 & 0.012 & 0.72 & 0.014 & 0.73 & 0.013 & 0.73 & 0.205 & 0.72 & 0.195 & 0.73 & 0.200 & 0.72 \\
\hline OP4 & 2 & 0.008 & 0.63 & 0.010 & 0.65 & 0.009 & 0.64 & 0.168 & 0.63 & 0.160 & 0.64 & 0.164 & 0.63 \\
\hline OP5 & 3 & 0.009 & 0.70 & 0.007 & 0.70 & 0.008 & 0.70 & 0.198 & 0.69 & 0.173 & 0.70 & 0.187 & 0.70 \\
\hline OP6 & 2 & 0.002 & 0.59 & -0.002 & 0.59 & 0.001 & 0.59 & 0.158 & 0.58 & 0.126 & 0.59 & 0.143 & 0.59 \\
\hline OP7 & 3 & 0.009 & 0.69 & 0.007 & 0.70 & 0.008 & 0.70 & 0.172 & 0.72 & 0.203 & 0.71 & 0.187 & 0.71 \\
\hline OP8 & 2 & 0.001 & 0.58 & -0.003 & 0.59 & -0.001 & 0.58 & 0.112 & 0.59 & 0.144 & 0.59 & 0.128 & 0.59 \\
\hline
\end{tabular}
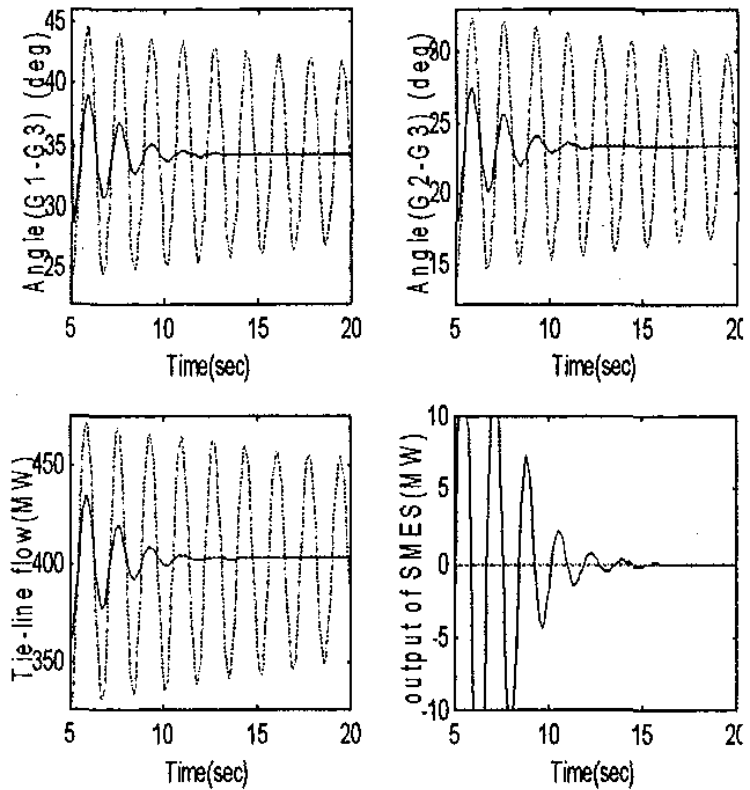

Fig. 6. Dynamic response of the system following a 3-phase fault at bus\#8. 400 MW power flow from area 1 to area 2 . Solid line: response with controller. Dotted line: response without controller.

\section{SMES SIZING}

The sizing of a SMES device requires the evaluation of both power and encrgy ratings. The power rating helps determine the nominal voltage and current of the coil and the nominal current of the converters. From the output response of the SMES in Fig. 6, it is seen that the real power output of the SMES oscillates between a $\pm 10 \mathrm{MW}$ limits for the first 5-8 $\mathrm{s}$ after the fault is applied. Following this period, the SMES becomes very effective in damping oscillations. Positive output indicates discharging of the coil whereas negative output indicates charging. The EPRI feasibility studies on energy storage applications suggest that energy rating be evaluated by integrating the output power traces of the SMES [22]. Energy involved
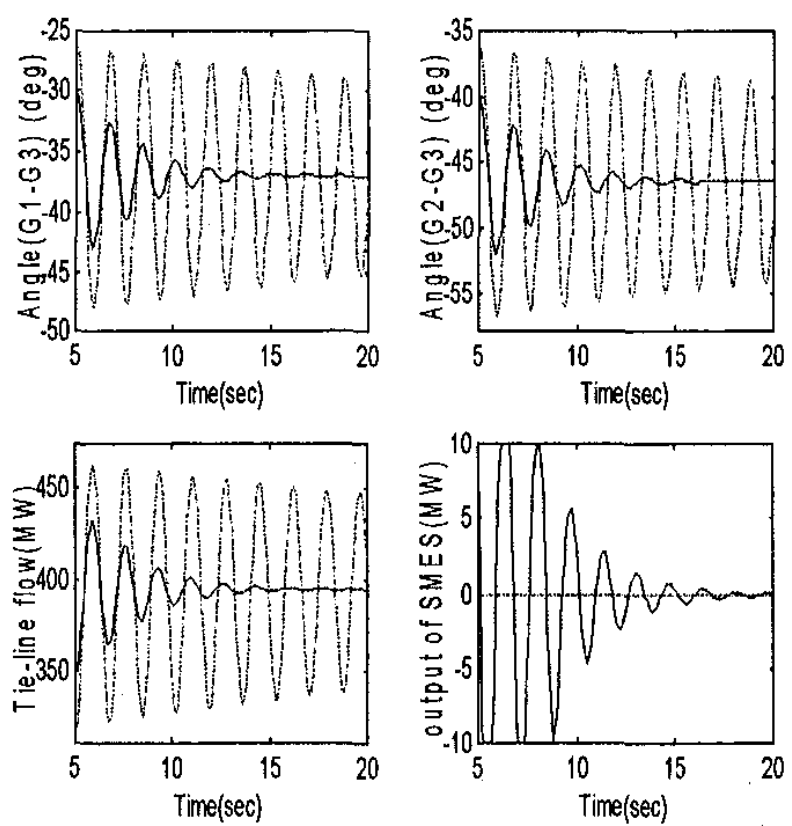

Fig. 7. Dynamic response of the system following a 3-phase fault at bus\#8. $400 \mathrm{MW}$ power flow from area 2 to area 1 . Solid line: response with controller. Dotted line: response without controller.

during charging and discharging are estimated by computing the area under each individual charging and discharging cycle. Assuming a turn around efficiency [22] of $100 \%$, it can be said that MW-second stored is the MW-second available for the SMES. With this assumption, the absolute value of the largest individual area (charging or discharging) can be decided as the energy rating in MJ when power is expressed in MW.

The energy evaluation approach just described is applied to SMES power traces in Figs. 5 and 6. It is found that an energy rating of $9 \mathrm{MJ}$ will be adequate to provide the desired damping performance. A $10 \mathrm{MW}, 9 \mathrm{MJ}$ SMES coil is sufficiently smaller than the first SMES coil (30 MJ) commissioned by BPA for damping oscillations in the Pacific AC intertie [23] in the early 
1980's. However, the technology for the BPA's 30 MJ coil was based on liquid helium cooled low temperature superconductor which was very costly. High temperature superconducting coils (HTSC) with liquid nitrogen as coolant are available commercially with low (1-3 MJ) energy rating. But recent trends in technology developments and demonstrations [24] for micro SMES $(1-10 \mathrm{MJ})$ in the USA under the initiatives of Department of Electronics (DOE), defense establishments and various industrial houses are very encouraging. It is highly likely that a 10 MJ SMES coil will be commercially available in the near future.

\section{CONCLUSIONS}

The work reported in this paper leads to several important conclusions regarding the damping control of the inter-area mode. They are listed as follows:

- A suitable controller location and an effective control signal are very impottant for the damping of the inter-area mode of oscillation.

- The frequency and time domain (transient response) specifications can be taken into consideration in the LMI formulation.

- The weight selection is less difficult than the Riccati based approach as the LMI based formulation does not impose restrictions on the location of the open-loop plant zeros.

- Even though a closed form analytical solution does not exist, numerically, the corresponding LMI can always be solved efficiently if it is feasible.

- The controller maintains acceptable damping for widely varying operating conditions which is very important from the operational perspective of any practical power system. The robustness of the controller is verified through nonlinear simulations for different operating scenarios.

- This test system has only one inter-area mode and two local modes. The proposed controller does not interfere adversely with the damping of local modes. In large interconnected power utilities, the existence of several FACTS devices is common and interactions among them through controller modes and multiple inter-area modes pose greater challenges in controller design. The proposed technique has been applied to design robust damping controllers for a relatively large system with four critical inter-area modes and eleven local modes. Three SMES devices are installed at three key locations. Non-linear simulation shows that controller based modal interactions are absent. However, we are planning to communicate the detail of our investigations for the large system in a separate paper.

- The proposed method can be applied for the design of damping controller with other FACTS devices.

\section{APPENDIX A}

Various operating conditions: Table III lists loads and generations for various operating conditions.

SMES Data: The symbols used in Fig. 2 have the following values. $T_{c p}=T_{c q}=0.026 \mathrm{~s}, K_{v}=10.0, T_{v 1}=1.0 \mathrm{~s}$,
TABLE III

GianIiRaTions AND I OADS IOR VARIOUS OPERATING CONDITIONS

\begin{tabular}{|c|c|c|c|c|}
\hline $\begin{array}{c}\text { Opera } \\
\text { ting } \\
\text { points } \\
(\mathrm{OP})\end{array}$ & $\begin{array}{c}\text { No of } \\
\text { Tie } \\
\text { Lines }\end{array}$ & $\begin{array}{c}\text { Power } \\
\text { flow(MW) }\end{array}$ & $\begin{array}{c}\text { Areal(A1) } \\
\text { Gen/Load(MW) }\end{array}$ & $\begin{array}{c}\text { Area2(A2) } \\
\text { Gen/Load(MW) }\end{array}$ \\
\hline 1 & 3 & 0 & $1227 / 1200$ & $1200 / 1180$ \\
\hline 2 & 2 & 0 & $1227 / 1200$ & $1200 / 1180$ \\
\hline 3 & 3 & $200(\mathrm{Al} \rightarrow \mathrm{A} 2)$ & $1420 / 1200$ & $1221 / 1400$ \\
\hline 4 & 2 & $200(\mathrm{~A} 1 \rightarrow \mathrm{A} 2)$ & $1420 / 1200$ & $1221 / 1400$ \\
\hline 5 & 3 & $400(\mathrm{~A} 1 \rightarrow \mathrm{A} 2)$ & $1440 / 1000$ & $1230 / 1600$ \\
\hline 6 & 2 & $400(\mathrm{~A} 1 \rightarrow \mathrm{A} 2)$ & $1440 / 1000$ & $1240 / 1600$ \\
\hline 7 & 3 & $400(\mathrm{~A} 2 \rightarrow \mathrm{A} 1)$ & $1230 / 1600$ & $1443 / 1000$ \\
\hline 8 & 2 & $400(\mathrm{~A} 2 \rightarrow \mathrm{A} 1)$ & $1230 / 1600$ & $1448 / 1000$ \\
\hline
\end{tabular}

$T_{v 2}=10.0 \mathrm{~s}, T_{v m}=0.02 \mathrm{~s}, P_{\mathrm{SM}, \max }=0.1, P_{\mathrm{SM}, \min }=-0.1$, $Q_{\mathrm{SM}, \max }=0.25, Q_{\mathrm{SM}, \min }=-0.25$.

\section{APPENDIX B}

Change of controller variable and substitution to matrix inequalities in (7) and (9) in Section III.

Consider inequalities (7) and (9) of Section III. Let $X$ and $X^{-1}$ be partitioned as

$$
X=\left(\begin{array}{cc}
R & M \\
M^{T} & *
\end{array}\right) \quad \text { and } \quad X^{-1}=\left(\begin{array}{cc}
S & N \\
N^{T} & *
\end{array}\right)
$$

where $R$ and $S$ are $n \times n$ symmetric matrices and " $*$ " denotes an expression not relevant for the present purpose. From the identity $X X^{-1}=I$, it can be inferred that

$$
X=\left(\begin{array}{c}
S \\
N^{T}
\end{array}\right)=\left(\begin{array}{l}
I \\
0
\end{array}\right)
$$

which leads to $X \prod_{2}=\prod_{1}$ with

$$
\prod_{1}:=\left(\begin{array}{cc}
R & I \\
M^{T} & 0
\end{array}\right), \quad \prod_{2}:=\left(\begin{array}{cc}
I & S \\
0 & N^{T}
\end{array}\right) .
$$

The new controller variables are defined as [17]:

$$
\begin{gathered}
\hat{A}=N A_{k} M^{T}+N B_{k} C_{2} R+S B_{2} C_{k} M^{T} \\
+S\left(A+B_{2} D_{k} C_{2}\right) R \\
\hat{B}=N B_{k}+S B_{2} D_{k} \\
\hat{C}=C_{k} M^{T}+D_{k} C_{2} R \\
\hat{D}=D_{k} .
\end{gathered}
$$

If $M$ and $N$ are of full row rank and if $\hat{A}, \hat{B}, \hat{C}, \hat{D}, R$, and $S$ are given, the controller variable $A_{k}, B_{k}, C_{k}$, and $D_{k}$ can be computed from (13)-(16). Moreover, if $M$ and $N$ are square $(k=n)$ and invertible matrices, then $A_{k}, B_{k}, C_{k}$, and $D_{k}$ are unique. For full order controller design, one can always assume that $M$ and $N$ have full row rank. Hence the variables $A_{k}, B_{k}, C_{k}$, and $D_{k}$ can always be replaced by $\hat{A}, \hat{B}, \hat{C}$, and $\hat{D}$, without loss of generality.

Now pre-multiplying and postmultiplying the inequality $X>0$ by $\prod_{2}^{T}$ and $\prod_{2}$, inequality (7) by diag) $\left.\prod_{2}^{T}, I, I\right)$ and $\operatorname{diag}\left(\Pi_{2}, I, I\right)$, inequality (9) by $\Pi_{2}^{T}$ and $\Pi_{2}$, and performing the necessary change of controller variables as defined in 
(13) $-(16)$, the LMI's in $(10)-(12)$ are obtained [17]. The variables in (10)-(12) are given by

$\Psi_{11}=\left[\begin{array}{cc}A R+R A^{T}+B_{2} \hat{C}+\hat{C}^{T} B_{2}^{T} & B_{1}+B_{2} \hat{D} D_{21} \\ \left(B_{1}+B_{2} \hat{D} D_{21}\right)^{T} & -\gamma I\end{array}\right]$

$\Psi_{21}=\left[\begin{array}{cc}\hat{A}+\left(A+B_{2} \hat{D} C_{2}\right)^{T} & S B_{1}+\hat{B} D_{21} \\ C_{1} R+D_{12} \hat{C} & D_{11}+D_{12} \hat{D} D_{21}\end{array}\right]$

$\Psi_{22}=\left[\begin{array}{cc}A^{T} S+S A+\hat{B} C_{2}+C_{2}^{T} \hat{B}^{T} & \left(C_{1}+D_{12} \hat{D} C_{2}\right)^{T} \\ C_{1}+D_{12} \hat{D} C_{2} & -\gamma I\end{array}\right]$

where

$$
\Phi=\left(\begin{array}{cc}
A R+B_{2} \hat{C} & A+B_{2} \hat{D} C_{2} \\
A & S A+\hat{B} C_{2}
\end{array}\right) .
$$

\section{REFERENCES}

[1] F. R. Schleif and J. H. White, "Damping for the northwest-southwest tic line oscillations-An analog study," IEEE Trans. on Power Apparatus and Systems, vol. PAS-85, no. 12, pp. 1239-1247, Dec. 1966.

[2] W. Fairney, A. Myles, T. M. Whitelegg, and N. S. Murray, "Low frequency oscillations on the $275 \mathrm{kV}$ interconnectors between Scotland and England," CIGRE, Paris, paper 31-08, Sept. 1982.

[3] M. Klein, G. J. Rogers, and P. Kundur, "A fundamental study of inter-area oscillations in power systcms," IEEE Trans. on Power Systems, vol, PWRS-6, no. 3, pp. 914-921, Aug. 1991.

[4] M. Klein, L. X. Le, G. J. Rogers, S. Farrokhpay, and N. J. Balu, " $H H_{\infty}$ damping controller design in large power systems," IEEE Trans, on Power Systems, vol. 10, no, 1, pp. 158-166, Feb. 1995.

[5] G. N. Taranto and J. H. Chow, "A robust frequency domain optimization technique for tuning series compensation damping controllers," IEEE Trans, on Power System, vol. 10, no. 3, pp. 1219-1225, Aug. 1995.

[6] G. N. Taranto, J. K. Shiau, J. H. Chow, and H. A. Othman, "Robust decentratised design of multiple FACTS damping controllers," in IEF Proc:-Generation, Transmission and Distribution, vol. 144, Jan. 1997, pp. $61-67$.

[7] J. Sefton and K. Glover, "Pole/zero cancellations in the general $H_{\infty}$ problem with reference to a two block design," Systems \& Control Let ters, vol. 14, pp. 295-306, 1990.

[8] L. Rouco, F. L. Pagola, A. Garcia-Cerrada, J. M. Rodriguez, and R. M. Sanz, "Damping of electromechanical oscillations in power systems with superconducting magnetic energy storage systems-Location and controller design," in Proc. of 12th Power System Computation Conference, Dresden, Aug. 19-23, 1996, pp. 1097-1104.

[9] H. A. Peterson, N. Mohan, and R. W. Boom, "Superconductive energy storage inductor-converter units for power systems," IEEE Trans. on $P A S$, vol. PAS 94, no. 4, pp. 1337-1348, 1975.

[10] Y. Mitani, K. Tsuji, and Y. Murakami, "Application of SMES to improve power system dynamic performance," IEEE Trans. on Power Systems, vol. 3, no. 4, pp. 1418-1425, 1988

[11] N. Martin and L. T. G. Lima, "Determination of suitable locations for power system stabilizer and static var compensators for damping electromechanical oscillations in large power systems," IEEE Trans. on Power Systems, vol. 5, no. 4, pp. 1455-1469, Nov. 1990.

[12] S. P. Boyd, L. El Ghaoui, E. Feron, and V. Balakrishnan, Linear Matrix Inequalities in Systems and Control Theory. Philadelphia, PA: SIAM, 1994.
[13] S. Skogestad and I. Postlethwaite, Multivariable Feedback Control Analysis and Design: John Wiley and Sons, 1996.

[14] K. Zhou, J. Doyle, and K. Glover, Robust and Optimal Control: PrenticeHall, 1995 .

[15] P. Gahinet and P. Apkarian, "A linear matrix inequality apptoach to $H_{\infty}$ control," International Journal of Robust and Nonlinear Control, vol. 4, pp. 421-448, 1994.

[16] M. Chilali and P. Gahinet, " $H_{\infty}$ design with pole placement constraints: An LMI approach," IEEE Trans. on Automatic Control, vol. 41, no. 3 , pp. 358-367, Mar. 1996.

[17] C. Scherer, P. Gahinet, and M. Chilali, "Multiobjective output-feedback control via LMI optimization," IEEE Trans. on Automatic Control, vol, 42, no. 7, pp. 896-911, July 1997.

[18] Y. Nesterov and A. Nemirovski, Interior Point Polynomial Methods in Convex Programming: Theory and Applications. Philadelphia, PA: SIAM, 1994.

[19] D. J. Limebeer and M. Green, Linear Robust Control: Prentice-Hall, 1995.

[20] P. Gahinet, A. Nemirovski, A. J. Laub, and M. Chilali, LMI Control Toolbox for Use with Matlab: The Math Works Inc, 1995.

[21] R. Y. Chiang and M. G. Sofonov, Robust Control Toolbox for Use with Matlab: The Math Works Inc., 1996

[22] R. J. Koessler, B. Fardanesh, M. I. Henderson, and R. Adapa, "Encrgy storage applications in New York State," in IEEE Power Engineering Society-FACTS Applications, 96TP $116-0$, pp. 8.46-8.51.

[23] J. D. Rogers, R. I. Schermer, B. L. Miller, and J. F. Hauer, "30-MJ superconducting magnetic energy storage system for electric utility transmission stabilization," Proc. of the IEEE, vol. 71, no. 9, pp. 1099-1107, Sept. 1983

[24] C. A. Luongo, "Superconductiing storage systems: An overview," WEEE Trans. on Magnetics, vol. 32, no. 4, pp. 2214-2223, July 1996.

Bikash C. Pal received his B.E.E. (honors) and M.E. degrees from Jadavpur University and the Indian Institute of Science in 1990 and 1992 respectively. Between 1992 and 1996 he worked with Tata Consulling Engineers, India and Jadavpur University, India. Currently, he is with the Imperial College, London, as a Ph.D. student.

Alun H. Coonick graduated from the University of Southampton in 1980 and obtained his Ph.D. degree in 1991. He is a Lecturer in the Department of Electrical and Electronic Engineering, Imperial College. His research interests include power system stability and control, using FACTS devices and artificial intelligence applied to power systems.

Imad M. Jaimoukha graduated from the University of Southampton in 1983 and obtained his $\mathrm{Ph} . \mathrm{D}$. degree in 1990 from Imperial College. He is a I.ecturet in the Department of Electrical and Electronic Engineering, Imperial College. His rescarch interests include robust multivariable control and model reduction.

Haitham Fl-Zobaidi graduated from the College of Engineering, University of Baghdad. He is currently working toward the Ph.D. degree at Imperial College. His research interests include multivariable control and model reduction. 